\title{
Geospatial clustering of meningitis: an early warning system (hotspot) for potential meningitis outbreak in upper east region of Ghana
}

\author{
Ernest Akyereko $^{1,2}$, Donne Ameme ${ }^{1}$, Kofi M. Nyarko ${ }^{1}$, Franklin Asiedu-Bekoe ${ }^{2}$ Samuel Sackey $^{1}$, Kofi \\ Issah $^{3}$, Baba Wuni ${ }^{4}$ and Ernest Kenu ${ }^{1,2}$
}

Ghana Med J 2020; 54(2) supplement: 32-39 DOI: http://dx.doi.org/10.4314/gmj.v54i2s.6

\author{
${ }^{1}$ Ghana Field Epidemiology and Laboratory Training Program, School of Public Health, University of Ghana, \\ Accra \\ ${ }^{2}$ Disease Surveillance Department, Ghana Health Service, Accra, Ghana \\ ${ }^{3}$ Brong Ahafo Regional Health Directorate, Ghana Health Service, Sunyani, Ghana \\ ${ }^{4}$ Upper East Regional Health Directorate, Ghana Health Service, Bolgatanga, Ghana
}

\author{
Corresponding author: Ernest Kenu \\ E-mail: ernest_kenu@yahoo.com
}

Conflict of interest: None declared

\begin{abstract}
SUMMARY
Objective: We mapped and generated hot spots for potential meningitis outbreak from existing data in Upper East region, Ghana.

Design: This was a cross-sectional study conducted in 2017

Data Source: Meningitis data in the Upper East Region from January 2007, to December 2016.

Main outcome measure: We used spatial tools in Quantum Geographic Information System (QGIS) and Geoda to draw choropleth map of meningitis incidence, case fatality and hotspot for potential meningitis outbreak

Results: A total of 2312 meningitis cases (suspected and confirmed) were recorded from 2016-2017 with median incidence of $15.0 \mathrm{cases} / 100,000$ population $(\min 6.3, \max 47.8$ ). Median age of cases was 15 years (IQR: 6-31 years). Most (44.2\%) of those affected were 10 years and below. Females $(51.2 \%)$ constituted the highest proportion. Median incidence from 2007-2011 was 20cases/100,000 population (Min 11.3, Max 39.9) whilst from 20122016 was 11.1 cases/100,000 populations (Min 6.3, Max 47.8). A total of 28 significant hotspot sub-districts clusters $(\mathrm{p}=0.024)$ were identified with 7 High-high risk areas as potential meningitis outbreak spots.

Conclusion: The occurrence of meningitis is not random, spatial cluster with high -high-risk exist in some sub-districts. Overall meningitis incidence and fatality rate have declined in the region with district variations. Districts with high meningitis incidence and fatality rates should be targeted for intervention.
\end{abstract}

Keywords: meningitis outbreak, geospatial clustering, Upper East Region, meningitis incidence, Ghana Funding: Author EA was supported by the West Africa Health Organization (Ref.: Prog/A17IEpidemSurveillN $\left.{ }^{\circ} 57212014 / \mathrm{mcrt}\right)$.

\section{INTRODUCTION}

Meningitis is an inflammation of the protective membranes of the brain and spinal cord (meninges). It is caused mainly by bacteria and viruses, but can also result from injuries, cancers, or certain drugs. Bacterial meningitis has great social and health impact due to its ability to cause outbreaks leading to sequelae and death. ${ }^{1}$ Several bacteria have the potential of causing meningitis but the common ones include Neisseria meningitidis (serogroups A, W135, C, X) and Streptococcus pneumoniae or Hemophilic influenzae type $B$.

Mechanisms for transmission of the infection is mainly from infected person to uninfected person by airborne droplets of respiratory or throat secretions. ${ }^{2}$ Infected person may show no sign and symptoms and yet carries Neisseria meningitidis unknowingly and may serve as important source for transmission. $^{3}$
Meningitis generally occurs 1-10 days after exposure. ${ }^{4}$ Meningitis has many symptoms, the common ones include a severe headache, high temperature, neurological signs such as lethargy, coma and /or convulsions, nausea and vomiting, Sensitivity to light (photophobia) and stiff neck. Infants and children aged $<2$ years, may have nonspecific symptoms. Neck stiffness, which is usually seen in meningitis cases, may be absent in this age group but may also show irritability, bulging fontanel with no neck stiffness.

Meningitis epidemics occur globally and pose major public health challenges despite the availability of safe and cost-effective vaccine. ${ }^{5,6}$ The global mortality rate for meningitis continues to be high, ranging between $2 \%$ to $30 \%$ notwithstanding the progress made in treating the condition. ${ }^{7}$ 


\section{Original Article}

The incidence of bacterial meningitis is higher in developing countries and children bear the highest consequence. ${ }^{1}$

In Africa, meningitis is endemic within the African meningitis belt which includes 14 countries stretching from Senegal to Ethiopia. Approximately, over 300,000 million people within the belt are at risk of bacterial meningitis morbidity and mortality. $2,6,8$

In Ghana, the Upper East, Upper West and Northern Regions fall within the "African meningitis belt" with high annual meningitis incidence, frequent outbreaks and death., ${ }^{2,9}$ The main strategy for meningitis prevention and control in Ghana includes surveillance and reactive vaccination of affected areas. ${ }^{10}$ Pneumococcal and proactive meningitidis A vaccines were introduced in 2012 and 2016 respectively for children below one year of age. The incidence of bacterial meningitis has been associated with environmental conditions and the incidence differs with respect to place. ${ }^{6}$ It is believed to mostly occur during the hot and dry season and drops when the rainy season begins. ${ }^{11}$

Geographic information system (GIS) has been helpful in providing a better understanding of a number of disease outcomes. ${ }^{12}$ GIS has shown its significance in examining spatial and temporal relationship between respiratory syndrome in pigs and human Streptococcus sui infection in Vietnam. ${ }^{13}$ In Ghana, a study in Suhum Kraboa Coaltar and Akuapem South Districts in Eastern Region used spatial tool to identify risk factors for Buruli ulcer in the districts. ${ }^{14}$ However, much exploration has not been done on meningitis incidence hot spot in Upper East Region using GIS. We generated hotspots maps for possible meningitis outbreak, spatio-temporal incidence and case fatality rates for meningitis in Upper East Region.

\section{METHODS}

\section{Study Area}

The Upper East Region is positioned in the north-eastern corner of Ghana, between longitude $0^{0}$ and $1^{0}$ West and latitudes $10^{0} 30^{\prime} \mathrm{N}$ and $11^{\circ} \mathrm{N}$. It shares borders with Burkina Faso to the north, the Upper West Region of Ghana to the west, the Republic of Togo to the east and the Northern Region of Ghana to the south (Figure 1). Upper East has a total land area of 8,842 sq. $\mathrm{km}$, which is about 2.7 percent of the total land area of Ghana.

Upper East Region has a population 1,046,545, with an estimated annual growth rate of 1.2 percent. The region has population density of 118.4 persons per square kilometer.

The climate of the Upper East Region is characterized by a bimodal rainy season from May/June to September/October. The rainfall is erratic spatially and in duration, with average annual rainfall ranging between $800 \mathrm{~mm}$ and $1.100 \mathrm{~mm}$ in the rainy season
The region experiences a long dry season from November to February, with dry, cold, and dusty harmattan winds. Average temperatures can be as low as 14 degrees at night during the dry season but may go above 35 degrees during the day. Relative humidity is low in Upper East Region

The region has eighty-sixed (86) health sub-districts with 297 health facilities. It also falls within the "Meningitis Belt" of Africa and onchocerciasis zone.

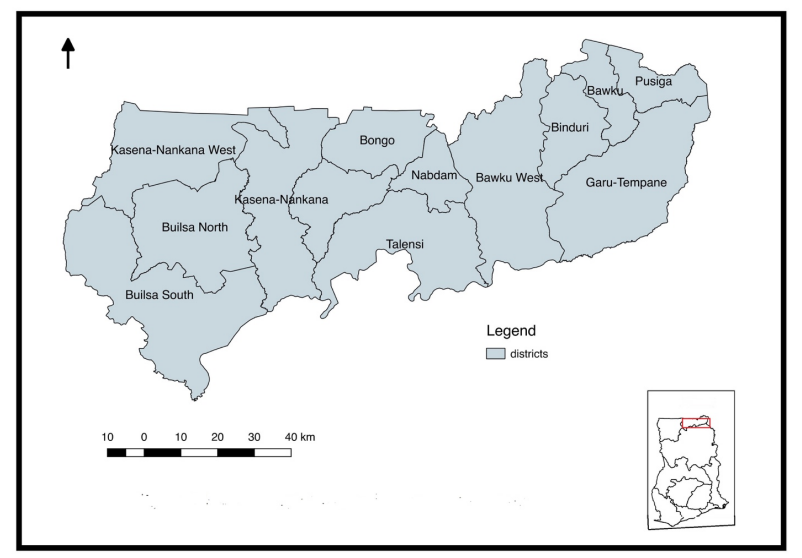

Figure 1 District map of Upper East Region Study Design

This was a geospatial analysis in which spatial tools in Quantum Geographic Information System (QGIS) and Geoda was used to perform a choropleth map of meningitis incidence and case fatality as well as clusters using 10 year secondary data on bacterial meningitis in the Upper East Region from January 2007 to December 2016.

\section{Study Population}

The target population for the study was made up of data on reported cases of meningitis from 2007 to 2016 in Upper East Region. This included weekly Integrated Disease Surveillance Responses (IDSR) reports, annual reports, line list, and outbreak reports within the period.

\section{Data Abstraction and Management}

Meningitis incidence data: Monthly and weekly Integrated Disease Surveillance and Response (IDSR) reports from the thirteen (13) districts within the study period were obtained. Meningitis incidence data for Upper East Region covering a period of 10 years stretching from 2007 to 2016 was extracted.

Key data elements like number of cases, age and sex distribution, number of deaths, date of onset, community/ village of occurrence, district of occurrence, and geographical position coordinates of incidence locations (district and sub-district of resident) were abstracted. The data were then entered into Microsoft Excel version 2016 and saved in comer separated value $(\mathrm{CSV})$ format. 


\section{Original Article}

Population data: The population data of Upper East Region (UER) for the year 2007 to 2016 was obtained from the Ghana Statistical Services Department 2010 population and housing report and excel was used to estimate the population from 2007 to 2016 using the regional growth rate of $2.1 \%$.

Spatial data preparation and analysis: A digitized boundary map of Ghana showing the regions, and districts (Ghana administration level 2) was used as a base map. We imported the Ghana Map into Quantum Geographic Information system (QGIS) ${ }^{15}$ and extracted the Upper East regional map.

Microsoft Excel (version 2016) was used to calculate the yearly cumulative incidence of cases per 100,000 population and case fatality rate for the years. The cumulative incidence and case fatality rates were presented as tables. Also, the excel file containing both the cases for each district per year and the population data was imported into the QGIS and joined to the Upper East regional map shapefile. The symbology was set to graduated symbol and the symbol method was set to colour. The classification was set to five classes at a precision of one. This was then used to generate a choropleth map showing high and low areas incidence and their respective case fatality rates.

Hotspot map: Spatial cluster was examined using Local Moran's I statistics in Geoda software. ${ }^{16}$ The spatial unit for the investigation was reduced to the sub-district level. This is to provide a risk map that narrows the level of risk to a small unit to help provide a targeted response. A line list on reported meningitis cases from 2012 to 2016 were used for the hot spot map. This list is a table which contains information on each reported case. At the time of the study, line lists from 2007 to 2011 were unavailable.

Data on incidence location from 2012-2016 abstracted from Upper East regional surveillance data was formatted to the sub-district level and joined to a digitized map showing subdistrict within the districts of Upper East region. Spatial weight was created by setting the weight manager at queen contiguity weight matrix and first order level was selected as the spatial neighbor at the sub-district level. Local Moran's I with Empirical Bayes rate (EBR) was used for the analysis. Therefore, Local Indicators of Spatial Association (LISA) displaying the presence or absence of significant spatial clusters and outliers for each sub-district was shown in a choropleth map showing areas of high risk and low risk.

The map was classified into high-high areas which will be the hottest spot, Low-high areas which are low risk areas surrounded by high risk areas, High-low areas which show high risk areas surrounded by low risk areas, Low-low which are low risk areas surrounded by low risk and No significant clusters which show areas of spatial randomness in the cases.
Ethical consideration: Ghana Health Service Ethical Review Committee approved the study (GHS-ERC 46/12/16). Permission was also sought from the Upper East Regional Director of Ghana Health Services. To ensure confidentiality and prevent any linkage., names of patients were coded with identification numbers All data abstracted for the study were stored in computers under passwords and hard copies (data abstraction forms) were kept under lock.

\section{RESULTS}

A total of 2312 meningitis cases (both suspected and confirm) and 313 (13.5\%) deaths were recorded within the tenyear period. The median age of the cases was 15 years (IQR: 6 to 31 years). The commonest age group affected was 10 years and below with about $44.2 \%\left(\chi^{2}=3.2, p=0.16\right)$ of the total cases. Among the cases $51.1 \%$ were female.

The annual median cumulative incidence of meningitis for the ten years was 15.9 cases $/ 100,000$ population. The highest incidence of cases was recorded in 2012 (Table 1) with incidence increasing up to $47.8 / 100,000$ population while the lowest incidence was recorded in 2015 with an incidence of $6.3 / 100,000$ population. The region recorded 3 confirmed outbreaks in Bongo District in 2010, Kasena-Nankana Municipality in 2012 and Builsa North District in 2012 within the 10-years. The median incidence for the first 5years (2007-2011) was 20 cases/ 100,000 population (Min 11.3 and Max 39.9) whilst the second five years (2012-2016) was 11.1 cases/100,000 populations (Min 6.3 and Max 47.8). From the 10-year trends, aside from 2016, there has been a general decline of meningitis incidence in the region.

Table 1 Incidence and case fatality rate for meningitis in Upper East region, 2007-2016

\begin{tabular}{|c|c|c|c|c|c|}
\hline Year & Cases & Death & $\begin{array}{l}\text { Population } \\
\text { at risk }\end{array}$ & $\begin{array}{l}\text { Incidence / } \\
100,000\end{array}$ & $\begin{array}{l}\text { Case fatal- } \\
\text { ity rate }\end{array}$ \\
\hline 2007 & 403 & 44 & $1,009,320$ & 39.9 & 10.9 \\
\hline 2008 & 158 & 36 & $1,021,579$ & 15.5 & 22.8 \\
\hline 2009 & 117 & 25 & $1,033,986$ & 11.3 & 21.4 \\
\hline 2010 & 404 & 62 & $1,046,545$ & 38.6 & 15.3 \\
\hline 2011 & 212 & 40 & $1,059,104$ & 20.0 & 18.9 \\
\hline 2012 & 512 & 49 & $1,071,813$ & 47.8 & 9.6 \\
\hline 2013 & 135 & 21 & $1,212,714$ & 11.1 & 15.6 \\
\hline 2014 & 87 & 7 & $1,227,443$ & 7.1 & 8.0 \\
\hline 2015 & 78 & 8 & $1,242,352$ & 6.3 & 10.3 \\
\hline 2016 & 206 & 21 & $1,257,441$ & 16.4 & 10.2 \\
\hline
\end{tabular}

The median case fatality rate for the 10 -year period was $13.1 \%$ (Min 8 and Max 22.8) with the highest recorded in $2008(22.8 \%)$ and the lowest in $2014(8.0 \%)$. The median case fatality rate for the first 5years (2007-2011) was $18.9 \%$ (Min10.9 and Max 22.8) whilst the second five years (20122016) was $10.2 \%$ (Min8 and Max 15.6).

The highest peak of the incidence was observed in 2012 . Most cases occurred from week 48 to week 20 . The increase starts from week 47 through to week 17. 
The highest peak occurred on Week 8 in 2012, Week 15 in 2013, Week 12 in 2014, Week 13 in 2015 and Week 10 in 2016 (Figure 2).

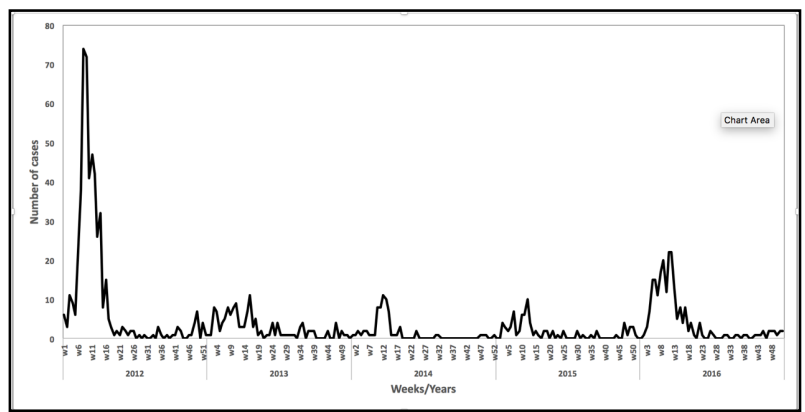

Figure 2 Meningitis weekly incidence in Upper East Region, 2012 to 2016

\section{Spatio-temporal incidence of meningitis in Upper East Region}

Cases of meningitis were reported in all district in Upper East region except Builsa South District in 2014, Binduri and Pusiga in 2013, Bawku West, Bawku Municipality, Binduri, and Pusiga in 2015 (Figures 3a \&b). For the 10-year period, the disease incidence was high in the western districts of the study area than the other districts. Areas such as KasenaNankana Municipality in 2008,2011,2015, Bongo District in 2010, 2011, 2013 and 2015, Bolgatanga Municipality in 2014, Builsa North in 2012, 2013, and Nabdam in 2014 recorded high incidence while Bawku West and Garu- Tempane recorded generally low incidence for the period. Comparing the first five years (Figure 3a) and the second five years (Figure $3 \mathrm{~b}$ ) incidence distribution among districts shows no major difference with the exception of Pusiga which recorded high incidence in 2016.

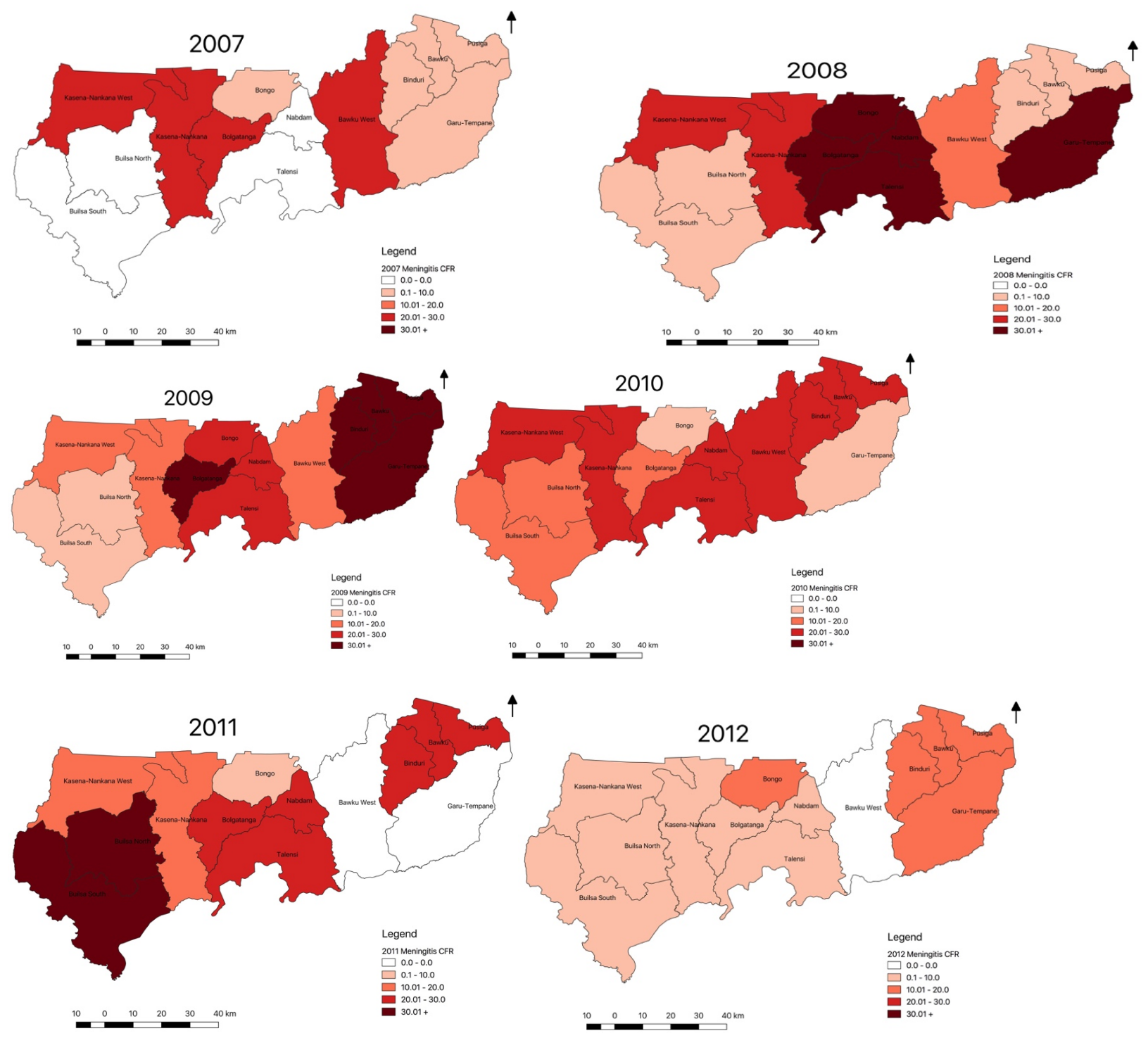

Figure 4a Spatio-temporal meningitis case fatality rate in districts of Upper East region from 2007 to 2012 


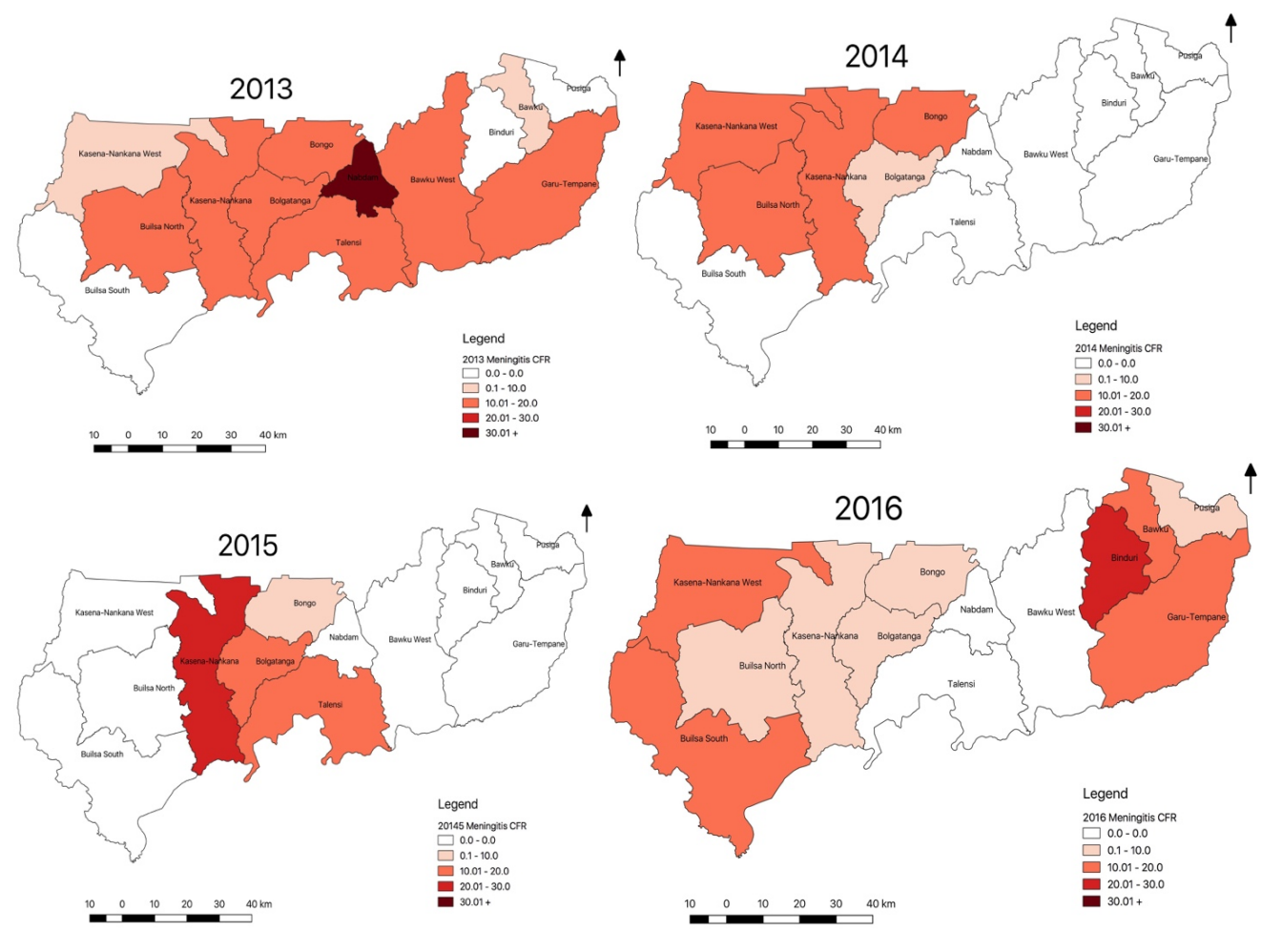

Figure 4b Spatio-temporal meningitis case fatality rate in districts of Upper East region from 2013 to 2016

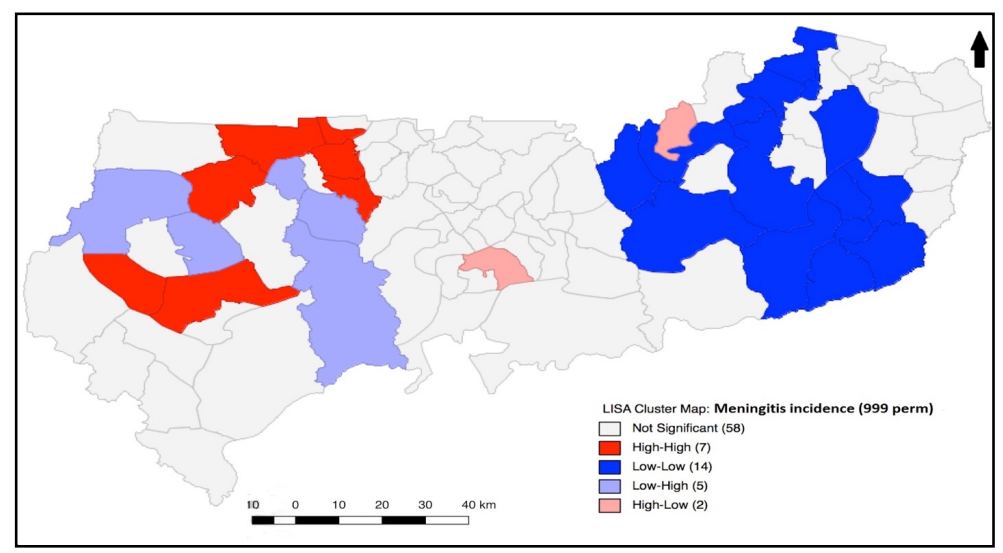

Figure 5 Weighted LISA cluster map showing hot spot for meningitis in sub-districts of Upper East region 2007-2016 (999perm, $\mathrm{p}=0.024$ )

\section{Spatio-temporal case fatality rate of meningitis in Up- per East Region}

The highest case fatality rate was observed in 2008 and 2009 with about five districts recording above $30 \%$ in each year whilst the least was recorded in 2015. These districts included five districts in 2008 (Bolgatanga, Bongo, Talensi, Nabdam and Garu-Tempane) and 2009 (Bolgatanga, Garu-Tempane, Pusiga, Bawku Municipality and Binduri). From 2007 to 2011, districts such as
Builsa North, Builsa South, Talensi, Nabdam in 2007, Bawku West and Garu-Tempani in 2011 recorded zero death from meningitis.

In the first five years, twelve (12) districts recorded case fatality rates above $30 \%$ whilst in the second five years only one district recorded above $30 \%$. All districts recorded death due to meningitis in 2008, 2009 and 2010. 


\section{Spatial Autocorrelation and Hotspot analysis}

Moran's I test used in the study identified a statistically significant positive at a global level $(p=0.024)$, which indicates that the meningitis incidence per 100,000 populations was too similar across neighboring sub-district and thus could not have occurred by chance. Also, local Moran's I test was employed at statistical significance level of $\alpha=0.05$ and 999 permutations as shown in Figure 5, identified positively correlated spatial sub-district cluster in Upper East region. There were 7 High-High meningitis risk hotspot sub-district, 15 Low-Low, 5 Low-High, 2 High-Low and 58 no significant cluster. The Eastern part of Upper East region covering Builsa North and KasenaNankana Municipality showed a significantly positive High-High $7(100 \%)$ with some Low-High while the Western part covering Garu-Tempane District, Binduri, and Bawku Municipality also show significant Low-Low 15 (100\%) (Table 2).

Table 2 Hot spot district and sub-district table

\begin{tabular}{|c|c|c|c|c|}
\hline Risk Level & Affected district & Sub-district & $\begin{array}{l}\text { LISA, I } \\
\text { value }\end{array}$ & P-values \\
\hline \multirow[t]{7}{*}{ High-High } & Kasena-Nakana Municipality & Navrongo East & 0.33 & $0.030^{*}$ \\
\hline & & Pungu & 1.80 & $0.009^{*}$ \\
\hline & & Paga & 2.01 & $0.020^{*}$ \\
\hline & Kasena-Nakana West & Chiana & 0.84 & 0.048 \\
\hline & & Navio & 0.34 & $0.03 *$ \\
\hline & Builsa North & Sandema & 1.75 & $0.006 *$ \\
\hline & & Siniensi & 0.69 & $0.02 *$ \\
\hline \multirow[t]{14}{*}{ Low-Low } & Binduri & Binduri central & 0.071 & $0.001 *$ \\
\hline & & Zawse-Bansi & 0.38 & $0.017 *$ \\
\hline & & Bazua & 0.39 & $0.031 *$ \\
\hline & & Goore-Manga & 0.33 & 0.05 \\
\hline & Bawku Municipality & Kaku/Zabugu & 0.071 & $0.002 *$ \\
\hline & & Urban East & 0.34 & 0.054 \\
\hline & & Urban West & 0.23 & $0.012 *$ \\
\hline & Garu-Tempane & Sango & 0.375 & $0.02 *$ \\
\hline & & Tempani & 0.291 & $0.02 *$ \\
\hline & & Garu & 0.304 & $0.04 *$ \\
\hline & & Worikambo & 0.23 & 0.05 \\
\hline & & Denugu & 0.32 & $0.043 *$ \\
\hline & Bawku west & Binaba & 0.38 & $0.003 *$ \\
\hline & & Tilli-Winnaba & 0.43 & 0.05 \\
\hline \multirow[t]{5}{*}{ Low-High } & Kasena-Nakana Municipality & Kolongo & -0.26 & $0.042 *$ \\
\hline & & Wuru & -1.54 & $0.001 *$ \\
\hline & & Vunania-kapania & -0.24 & $0.014 *$ \\
\hline & Builsa North & Sandema East & -0.26 & $0.001 *$ \\
\hline & Kasena-Nakana West & Kativ & -0.36 & $0.029 *$ \\
\hline \multirow[t]{2}{*}{ High-Low } & Talensi & Tongo & -0.45 & $0.03^{*}$ \\
\hline & Bawku west & Zabila North & -0.18 & $0.001^{*}$ \\
\hline
\end{tabular}

*significant clusters

\section{DISCUSSION}

The study identified 28 clusters $(\mathrm{p}=0.024)$ showing statistically significant hot spots in Upper East Region. Of the eighty-six (86) sub-districts in the region where the study was performed, the hottest spot was found in Navrongo East, Pungu and Paga which are located in KasenaNankana Municipality, Chiana and Navio which are found in Kasena-Nankana West District and Sandema, Siniesi in Builsa North District. Kasena- Nankana Municipality recorded the highest number of hot sub-district as shown in Figure 5. Incidentally Kasena-Nankana $\mathrm{Mu}-$ nicipality recorded an outbreak in 2012. Kasena Nankana Municipality is located at the upper part of the region thus may experience greatly the dry and dusty northeast trade winds which originates from the Sahara Desert. ${ }^{17}$ The difference in weather conditions across sub-districts may have resulted in the identified hotspots in Upper East Region.
Several studies have suggested that environmental variables have influence on meningitis incidence. ${ }^{8,10,18,19}$ Ayden and colleagues identified weather as responsible for explaining some of the differences in meningitis counts, using log-linear models. ${ }^{8}$ The study suggested that weather conditions can improve estimated average laboratory-confirmed cases of meningitis counts up to $40 \%$.

The study also showed a decreasing trend in meningitis incidence and cases fatality rate in the region for the 10 year period. The region recorded a median meningitis incidence of [15.9 cases/100,000 population (min 6.3 and $\max 47.8)]$. This could be due to the mass vaccination in 2012 and health education on meningitis over the years. The median incidence was lower than a review of surveillance data of countries within the meningitis belt by World Health Organization which saw a median attack rate of 109/100,000 (min-max 79-134). ${ }^{20}$ 
Moreover, meningitis surveillance data review by Kaburi in northern region of Ghana from 2010 to 2015 showed meningitis incidence ranging from 1.6 to $62.6 .{ }^{21}$

The incidence median of the last five years (2012-2017) was lower than the incidence median of the first five years (2007-2011). The highest incidence $(47.77 / 100,000)$ was recorded in 2012 during an outbreak that affected Kasena-Nankana Municipality and Builsa North Districts. This made the region to embark on reactive vaccination against $N$. meningitidis W135. The heightened vaccination could have been responsible for the low incidence recorded in 2014(7.9/100,000) and $2015(6.28 / 100,000)^{22}$ which may have caused the reduction in the median incidence in the second five years. On the contrary, $2016(16.38 / 100,000)$ saw an increase and this could be due to the fact that the reactive vaccine provides protection for two years and therefore the immunity level against meningitis may be decreasing making more people within the region susceptible. Currently, Ghana is has included Neisseria Meningitidis (MenAfriVac) vaccine and 13-valent pneumococcal polysaccharide-diphtheria $\mathrm{CRM}_{197}$ protein conjugate vaccine (PCV13) into the routine immunization programmes and it is expected to reduce further cases of meningitis in the country. ${ }^{23}$

The study also showed that the attack rate varies across districts. Districts such as Kasena-Nankana Municipality, Kasena-Nankana West District, Bongo and Builsa North recorded above 90/100,000 population. The highest incidence was recorded in Kasena-Nankana Municipality which recorded $187 / 100,000$ in 2012 during the outbreak. These places were also identified in the Moran's I as high-high rate clusters and as such potential places for possible meningitis outbreak. Moreover, Telensi district which is located in the southern part of the region which used to record lower incidence between the first five years (2007-2011) recorded high cases during the second half-year (2012-2016). This could be due to the changing pattern of weather conditions and other disease transmission dynamics.

The regional case fatality rate also shows a similar trend to that of the incidence. The median incidence and case fatality rate for the second five years (2012 to 2016) was lower than the first five years (2007 to 2011). The difference in mean incidence and mean case fatality rate for the first five years and second five years were found to be significant $(p<0.05)$. This reduction could be due to the mass vaccination in the region in 2012 as well as other factors such as improvement in health workforce, diagnostic capacity, and health care accessibility due to the provision of more CHPS compounds to detect cases early for appropriate response. The median case fatality rate of $13.1 \%$ (Range: 8 to 22.8 ) is consistent with WHO (2010) estimated case fatality $(10-20 \%)$ for the countries in the meningitis belt.
A review of surveillance data from 2010 to 2014 by Opare also saw a case fatality rate of $13 \%$ in Upper East Region whilst Kaburi and colleagues reported case fatality rates ranging from 0 to $33.3 \%$ in their evaluation of the enhanced meningitis surveillance system of Yendi Municipality in the Northern Region of Ghana. ${ }^{9,21}$ Yet this figure is not the same across the districts, the district case fatality rate ranges from zero to $39.4 \%$. This could be due to the difference in geographical accessibility to health facilities and also health seeking behavior of the residents in the sub-districts. Therefore, interventions targeted at reducing meningitis case fatality rate should target districts who recorded high case fatality rate like Binduri and Bawku West. Even though there were outbreaks in the area in $2010(15 \%)$ and $2012(9.6 \%)$, there was relatively low case fatality rates than the years without outbreaks. This could be as a result of high reported cases during that year which could reduce the overall case fatality rate and also because, during outbreaks, the public would be alerted because of the health education and other interventions and may report early to the hospital for management. Moreover, during outbreaks districts normally receive technical, logistic, drug and financial support from regional and national level which could help them reduce mortalities. The results show that there has been a significant decrease in case fatality rate comparing the mean case fatality rate for 2007-2011(19.6\% \pm $3.3 \mathrm{SD})$ and $2012-2016(11.0 \% \pm 3.2 \mathrm{SD})$. This could be due to improvement in health facilities and staff in the region.

Limitation of the study: The data described in the study was based on data reported to the Upper East Regional Health Service through its facilities in the various districts and sub-districts. These numbers may not be representative of actual numbers and distribution of bacterial meningitis cases in the Upper East region. Also since the cases were aggregated into administrative boundaries that may be large and heterogeneous across districts in the region, this may underscore the actual disease distribution. The generation of the hotspot map was limited to data from 2012 to 2016 because completed line list from 2007-2011 was not available to study but the five-year data was enough for the construction of the map

\section{CONCLUSION}

The Moran's I risk map generated shows that cases of meningitis do not occur at random, and thus spatial cluster was identified in 28 sub-districts. Areas of High-high, Low-low, High low, as well as Low-high meningitis risk levels, exist. Kasena-Nankana municipality was identified as the hottest spot for potential meningitis outbreak. Meningitis incidence decreased marginally whilst case fatality rate decreased rapidly for the 10 -year period but 
still continues to be a major challenge in the region. Spatial variation in incidence and case fatalities were observed across districts in Upper East Region. Districts such as Kasena-Nankana Municipality, Builsa North district, and Bongo district have high incidence meningitis incidence and require targeted intervention.

\section{ACKNOWLEDGEMENT}

We would like to thank staff of Upper East Regional Health directorate especially the disease control unit for their cooperation and support during the study. We would also like to thank GFELTP for their assistance and guidance in this research.

\section{REFERENCES}

1. de Souza SF, Costa M da CN, Paim JS, et al. Bacterial meningitis and living conditions. Rev Soc Bras Med Trop. 2012;45(3):323-328.

2. Agier L, Deroubaix A, Martiny N, Yaka P, Djibo A, Broutin H. Seasonality of meningitis in Africa and climate forcing: aerosols stand out. J R Soc Interface. 2013;10(79):20120814.

3. Bharti N, Broutin H, Grais RF, et al. Spatial dynamics of meningococcal meningitis in Niger: observed patterns in comparison with measles. Epidemiol Infect. 2012;140(8):1356-1365.

4. CDC. Meningococcal | Outbreaks | CDC. http://www.cdc.gov/meningococcal/outbreaks/index.html. Published 2016.

5. Christensen H, Hickman M, Edmunds WJ, Trotter CL. Introducing vaccination against serogroup B meningococcal disease: An economic and mathematical modelling study of potential impact. Vaccine. 2013;31(23):2638-2646.

6. Bassey BE, Vaz RG, Gasasira AN, et al. Pattern of the meningococcal meningitis outbreak in Northern Nigeria, 2009. Int J Infect Dis. 2016; 43:62-67.

7. Owusu M, Nguah SB, Boaitey YA, et al. Aetiological agents of cerebrospinal meningitis: a retrospective study from a teaching hospital in Ghana. Ann Clin Microbiol Antimicrob. 2012; 11:28.

8. Dukić V, Hayden M, Orgor AAF, Opson TH, Hopson $\mathrm{T}$ et al. The Role of Weather in Meningitis Outbreaks in Navrongo, Ghana: A Generalized Additive Modeling Approach. 2012;17(3):442-460.

9. Opare J, Awoonor-Williams J, Odoom J, et al. Bacterial Meningitis: A Review in the Upper East Region of Ghana 2010-2014. Int J Trop Dis Heal. 2015;10(3):1-11.

10. Paireau J, Girond F, Collard J. Analysing SpatioTemporal Clustering of Meningococcal Meningitis
Outbreaks in Niger Reveals Opportunities for Improved Disease Control. PLoS Negl Trop Dis. 2012;6(3).

11. Patz JA, Githeko AK, McCarty JP, Hussein S, Confalonieri U. Climate change and infectious diseases. Infect Dis. 2008;9(6):103-132.

12. Mobasheri M, Ahmadi A. Incidence Patterns and Spatial Analysis of the Most Common Cancers in Southeastern Iran Using Geographic Information System (GIS). Acad J Canc Res 2014;7(2):141-145.

13. Huong VTL, Thanh L V, Phu VD, et al. Temporal and spatial association of Streptococcus suis infection in humans and porcine reproductive and respiratory syndrome outbreaks in pigs in northern Vietnam. Epidemiol Infect. 2015;144(1):35-44.

14. Kenu E, Nyarko KM, Seefeld L, Ganu V. et al. Risk Factors for Buruli Ulcer in Ghana. A Case Control Study in the Suhum-Kraboa-Coaltar and Akuapem South Districts of the Eastern Region. PLoS Negl Trop Dis. 2014;8(11):1-8.

15. QGIS Development Team, 2016. QGIS Geographic Information System. Open Source Geospatial Foundation Project.

16. Anselin, Luc, Ibnu Syabri and Youngihn Kho (2006). GeoDa: An Introduction to Spatial Data Analysis. Geographical Analysis 38 (1), 5-22)

17. GSS, GHS and ICF Macro. Ghana Demographic and Health Survey 2014. Accra; 2015.

18. Nii S, Codjoe A, Nabie VA. Climate Change and Cerebrospinal Meningitis in the Ghanaian Meningitis Belt. Int J Environ Res Public Heal Int J Environ Res Public Heal. 2014;113390(10):6923-6939.

19. Sultan B, Labadi K, Guégan JF, Janicot S. Climate drives the meningitis epidemics onset in West Africa. PLoS Med. 2005;2(1):0043-0049.

20. WHO. Meningitis Outbreak Response in Sub-Saharan Africa. Geneva; 2014.

21. Kaburi BB, Kubio C, Kenu E, Nyarko KM. et al. Evaluation of the enhanced meningitis surveillance system, Yendi municipality, northern Ghana, 20102015. BMC Infect Dis. 2017;17(1):1-11.

22. WHO. WHO $\mid$ Meningitis vaccine provides hope to people in Ghana. WHO. 2014. http://www.who.int/features/2012/meningitis_ghana/en/. Accessed October 25, 2016.

23. Kwambana-Adams BA, Asiedu-Bekoe F, Sarkodie $\mathrm{B}$, et al. An outbreak of pneumococcal meningitis among older children ( $\geq 5$ years) and adults after the implementation of an infant vaccination programme with the 13-valent pneumococcal conjugate vaccine in Ghana. BMC Infect Dis. 2016; 16(1): 575. 